\title{
Determination of asteroid masses ${ }^{\star}$
}

\section{II. (6) Hebe, (10) Hygiea, (15) Eunomia, (52) Europa, (88) Thisbe, (444) Gyptis, (511) Davida and (704) Interamnia}

\author{
G. Michalak ${ }^{\star \star}$ \\ Wrocław University Observatory, Kopernika 11, 51-622 Wrocław, Poland \\ Received 29 August 2000 / Accepted 17 April 2001

\begin{abstract}
New masses of eight asteroids: (6) Hebe, (10) Hygiea, (15) Eunomia, (52) Europa, (88) Thisbe, (444) Gyptis, (511) Davida, and (704) Interamnia, were determined. In most cases, the masses were calculated by means of the least-squares method as the weighted means of the values found separately from the perturbations on several single asteroids. Encounters suitable for determination of masses of these asteroids were found from the extensive search for large asteroidal perturbations exerted by massive asteroids on 4500 numbered minor planets. Most of the encounters found within the search had never before been used for mass determinations. The masses of Hebe, Hygiea, Eunomia, Europa, Thisbe, Gyptis, Davida and Interamnia were determined from perturbations on, respectively, 2, 8, 3, 4, 1, 1, 2 and 3 asteroids. As an outcome of the search for possible perturbers among the 912 largest asteroids, we propose and use correct dynamical models including important perturbers for all asteroids under consideration. For all new asteroid masses, the influence of the variation of the masses of asteroids in the dynamical model is investigated. A discussion on individual mass determinations is also presented.
\end{abstract}

Key words. minor planets, asteroids - astrometry - planets and satellites: individual: 6 Hebe, 10 Hygiea, 15 Eunomia, 52 Europa, 88 Thisbe, 444 Gyptis, 511 Davida, 704 Interamnia

\section{Introduction}

In 1998, the Asteroid Mass Determination Program was initiated at the Wrocław University Observatory. Within the Program, a search for detectable perturbations exerted by the largest 250 asteroids on the orbits of the remaining 4500 was carried out. As a first result, we published new determinations of the masses of three large asteroids: Ceres, Pallas and Vesta (Michalak 2000, hereafter Paper I).

In this second paper we determine the masses of eight subsequent asteroids. These are (6) Hebe, (10) Hygiea, (15) Eunomia, (52) Europa, (88) Thisbe, (444) Gyptis, (511) Davida and (704) Interamnia. Searching for asteroids perturbed significantly by the above eight bodies, we found in total 109 useful candidates. In particular, we found 14 cadidates for the determination of mass of Hebe, 38 for Hygiea, 11 for Eunomia, 17 for Europa, 2 for Thisbe, 1 for Gyptis, 13 for Davida and 13 for Intermnia. All were used to determine the masses of the perturbing asteroids, but, as in Paper I, we accepted only solutions with the smallest formal errors.

\footnotetext{
* Tables 4 and 7 and Fig. 1 are only available in electronic form at http://www.edpsciences.org

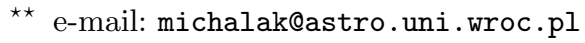

Table 1. The asteroids used in our standard dynamical model. Updated masses of Ceres, Pallas and Vesta were taken from Michalak (2000); the remaining masses are the same as those used by Viateau \& Rapaport (1998).

\begin{tabular}{ll}
\hline Asteroid & Mass [in $\left.10^{-10} M_{\odot}\right]$ \\
\hline (1) Ceres & 4.7 \\
(2) Pallas & 1.21 \\
(4) Vesta & 1.36 \\
(10) Hygiea & 0.47 \\
(11) Parthenope & 0.026 \\
(52) Europa & 0.14 \\
(511) Davida & 0.18 \\
(704) Interamnia & 0.35 \\
\hline
\end{tabular}

All methods used in this paper are described in detail in Paper I. The differences in comparison to Paper I are the following: (i) the current standard dynamical model is an updated one with the new masses of Ceres, Pallas and Vesta, taken from Paper I (Table 1), (ii) in addition, for each individual mass determination, the dynamical model was supplemented by inclusion of other asteroid(s) which could influence the orbit of the test asteroid (see Sect. 2 for explanation), (iii) the standard deviation of 
the mean masses were derived from the error propagation formula (Eq. (1) in Paper I) for all eight asteroids, owing to the small number of the test asteroids used in the mass determinations.

The main goal of this series of papers is to find perturbed test asteroids suitable for the mass determinations and also give correct dynamical models including important perturbing asteroids. The complete and reliable solution of the problem of asteroid masses requires simulataneous determination of a large number of asteroid masses. In such a solution one must use: (i) the correct dynamical model (including all important perturbing asteroids) and (ii) as complete as possible sets of observations. If either of the above two conditions is not fulfilled then a simplified method of mass determination, in which one mass is calculated from perturbations on one test asteroid, can be used. This is the case in this paper; due to uncertainty in masses of many asteroids in the dynamical models and incomplete sets of observations, obtained from Minor Planet Center (MPC) only, this method gives useful first approximations of the masses. This is a good base for future improvements, involving a large number of asteroids used in a simultaneous solution.

\section{The asteroid masses and densities}

A summary of the new mass determinations of the eight asteroids is given in Table 2. Table 3 details the postfit, post-selection $(\mathrm{O}-\mathrm{C})$ mean residuals for the standard dynamical model. The mean residuals for extended dynamical models-see below-are very similar. For each of the test asteroids used in the mass determination, the $(\mathrm{O}-\mathrm{C})$ residuals and details of the perturbations are given in Fig. 1. The residuals are calculated for the solutions involving the standard dynamical model. Blocks of observations, where selection and weighting was made independently, are marked with vertical lines. The perturbation plots show the differences in right ascension between perturbed and unperturbed orbits of the test asteroids in the case of backward integration starting from the epoch 1998 July 6.0 TDT, as well as the evolution of the mutual distance between the massive and the test asteroid.

In the least-squares adjustment, the initial masses of (10) Hygiea, (52) Europa, (511) Davida and (704) Interamania were the same as in the standard dynamical model (Table 1). The initial masses for the remaining four asteroids were determined from diameters given by Tedesco (1989) assuming a density of $3 \mathrm{~g} \mathrm{~cm}^{-3}$.

For the asteroids considered in this paper, the number of individual determinations is not large (see Table 2). One of the systematic effects (see Sect. 2.3 in Paper I) which can be relatively easily recognized and minimized is the incompleteness of the dynamical model. For this reason, we made a search for perturbers that can influence the orbits of all test asteroids used in this paper. As perturbers, we took 912 objects with diameters larger than $50 \mathrm{~km}$ (Tedesco 1989; Bowell et al. 1979; Tedesco 1992, diameters estimated from the absolute brightness). Some of the masses were taken from the literature and our preliminary determinations; the other ones were estimated from diameter assuming a density of $3 \mathrm{~g} \mathrm{~cm}^{-3}$. The results of the search are presented in Table 7 , in which all perturbers of the test asteroids giving a maximum perturbation effect in right ascension larger than $0.2^{\prime \prime}$, are listed. The search for perturbers was made in the same way as the search for encounters (see Sect. 2.2 in Paper I), i.e. included the backward integration from the epoch $1998 \mathrm{Jul}$. 6.0 TDT. This way of searching for possible perturbing asteroids favours those having relatively recent encounters. Neglecting perturbations smaller than $0.2^{\prime \prime}$ in RA acting at the beginning of the period covered by the observations is justified because noise in the observations at that epoch is about an order of magnitude larger than the neglected perturbations.

For calculation of perturbations in Table 7, asteroid masses given in Table 6 were used. If it happens in the future that some of the perturbing masses will appear to be significantly different from those we here assumed, the maximum perturbation given in Table 7 can be simply rescaled (multiplied by the $m / m_{0}$, where $m$ is the new mass and $m_{0}$ is the mass given in Table 6). This enables one to specify exactly which asteroids are important in the dynamical model.

The asteroid masses in Table 2 were calculated for two different dynamical models. In the column denoted "Mass STD model" the masses were calculated using the standard dynamical model (Table 1), while in the column "Mass EXT model", individual, extended dynamical models were applied. The extended dynamical models are the standard dynamical model supplemented with additional perturbing asteroids according to the results from Table 7. These models are the same as that denoted by "STD+ALL" in Table 4 (see below for explanation).

We also investigated the influence of inclusion of each of the additional perturbing asteroids on derived masses. The results are presented in Table 4, where for a variety of dynamical models we give: derived mass (its standard deviation was given only for the standard dynamical model denoted by "STD" since for the extended models it is practically the same), number of accepted observations (sum of observations in RA and Dec), global RMS error of the observations, and the difference $\Delta m$ between the current mass and that obtained when using the standard dynamical model. The difference is expressed in standard deviations of the mass calculated for the standard dynamical model.

For each test asteroid in Table 4, we first calculated the influence of the variations of the masses $(m \pm \sigma)$ included in the standard dynamical model. The standard deviation $\sigma$ of the mass of (1) Ceres, (2) Pallas and (4) Vesta were taken from Paper I (0.04, 0.26 and $0.05 \times 10^{-10} M_{\odot}$, respectively). For (10) Hygiea and (704) Interamnia the standard deviations were $0.23 \times 10^{-10} M_{\odot}$ and $0.17 \times 10^{-10} M_{\odot}$, respecively (see Table 1 in Paper I). For (52) Europa and (511) Davida, due to the fact that there were no previous determinations of their masses, 
Table 2. The results of the mass determinations for eight minor planets from perturbations on individual test asteroids. $\left|M_{\mathrm{p}}\right|$ is the largest perturbation effect in right ascension multiplied by $\cos \delta$, calculated for masses given in Table 6 . Residuals and details of the perturbations are given in Fig. 1. "STD" denotes standard dynamical model (Table 1), "EXT" denotes extended dynamical model (see Sect. 2, Tables 4 and 7 for more details). In the case of lack of additional perturbing asteroids in the extended dynamical models, the masses for the standard model were retained and used for calculation of the weighted mean of the mass.

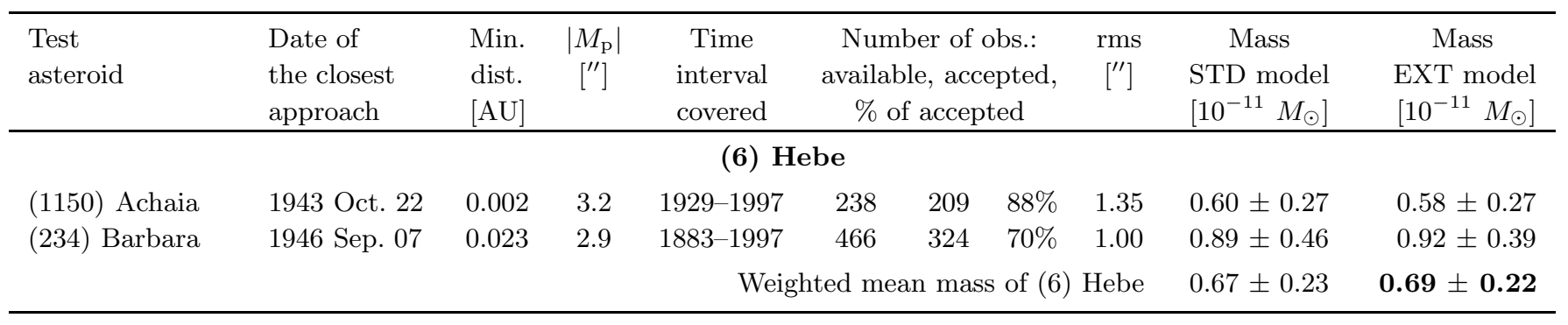
(7) Iris
(20) Massalia
(829) Academia
(111) Ate
(209) Dido
(69) Hesperia
(116) Sirona
(60) Echo

.

1928 Jan 18

1933 Nov. 05

$\begin{array}{llll}0.072 & 1.7 & 1848-1997 & 8110\end{array}$

1927 May 19

1878 Feb. 11

1958 Jan. 14

1951 Sep. 05

1939 May 21

1867 May 07

$\begin{array}{lll}0.150 & 2.9 & 1853-1997\end{array}$

2370

$\begin{array}{lll}7127 & 88 \% & 0.62\end{array}$

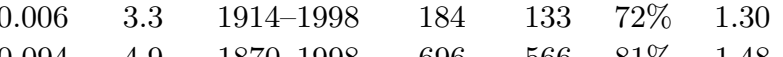

$\begin{array}{lllllll}0.094 & 4.9 & 1870-1998 & 696 & 566 & 81 \% & 1.48\end{array}$

$\begin{array}{lllllll}0.179 & 1.3 & 1879-1994 & 648 & 449 & 69 \% & 1.73\end{array}$

$\begin{array}{lllllll}0.086 & 1.9 & 1861-1997 & 876 & 635 & 72 \% & 1.69\end{array}$

$\begin{array}{lllllll}0.050 & 3.2 & 1872-1998 & 748 & 547 & 73 \% & 1.47\end{array}$

$\begin{array}{lllllll}0.211 & 3.0 & 1861-1997 & 860 & 636 & 74 \% & 2.15\end{array}$

Weighted mean mass of (10) Hygiea: Weighted mean without solution obtained from (7) Iris:

$\begin{array}{lr}4.90 \pm 0.70 & 8.96 \pm 0.66 \\ 4.55 \pm 1.26 & 4.25 \pm 1.26 \\ 5.47 \pm 1.56 & 5.90 \pm 1.44 \\ 8.24 \pm 1.81 & 7.24 \pm 1.80 \\ 4.29 \pm 2.46 & 5.98 \pm 2.25 \\ 5.24 \pm 2.47 & 5.49 \pm 2.50 \\ 5.16 \pm 2.58 & 5.94 \pm 2.58 \\ 6.33 \pm 2.66 & 5.80 \pm 2.68 \\ 5.21 \pm 0.50 & 7.35 \pm 0.48 \\ 5.53 \pm 0.71 & \mathbf{5 . 5 7} \pm \mathbf{0 . 7 0}\end{array}$

$4.90 \pm 0.70$

$8.96 \pm 0.66$

$4.25 \pm 1.26$

$7.24 \pm 1.80$

$5.98 \pm 2.25$

$5.49 \pm 2.50$

$5.94 \pm 2.58$

$7.35 \pm 0.48$

$5.53 \pm 0.71 \quad \mathbf{5 . 5 7} \pm \mathbf{0 . 7 0}$

\section{(15) Eunomia}

\begin{tabular}{llllllllllr} 
(1284) Latvia & 1960 Apr. 06 & 0.009 & 3.5 & $1925-1998$ & 264 & 198 & $75 \%$ & 1.17 & $1.25 \pm 0.34$ & $1.25 \pm 0.34$ \\
(1313) Berna & 1955 May 11 & 0.031 & 4.2 & $1911-1998$ & 154 & 137 & $89 \%$ & 1.06 & $1.29 \pm 0.60$ & $1.30 \pm 0.60$ \\
(7) Iris & 1873 Sep. 06 & 0.315 & 0.8 & $1848-1997$ & 8110 & 7191 & $89 \%$ & 0.61 & $1.33 \pm 0.65$ & $-1.83 \pm 0.64$ \\
& \multicolumn{1}{c}{ Weighted mean mass of (15) Eunomia: } & $1.27 \pm 0.27$ & $0.72 \pm 0.27$ \\
& \multicolumn{4}{c}{ Weighted mean without solution obtained from (7) Iris: } & $1.26 \pm 0.30$ & $\mathbf{1 . 2 6} \pm \mathbf{0 . 3 0}$
\end{tabular}

(993) Moultona

(1023) Thomana

(306) Unitas

(84) Klio
1966 Aug. 31

1971 May 31

1945 Jan. 14

1994 Mar. 09

\section{(52) Europa}

\begin{tabular}{|c|c|c|c|c|c|c|c|c|}
\hline 0.022 & 2.1 & 1928-1998 & 314 & 278 & $89 \%$ & 1.29 & $2.46 \pm 1.47$ & $2.46 \pm 1.47$ \\
\hline 0.007 & 4.2 & 1924-1998 & 178 & 150 & $84 \%$ & 2.10 & $1.79 \pm 1.55$ & $1.79 \pm 1.55$ \\
\hline 0.098 & 2.8 & 1892-1997 & 798 & 639 & $80 \%$ & 2.28 & $3.77 \pm 1.58$ & $3.63 \pm 1.58$ \\
\hline 0.150 & 3.5 & 1865-1998 & 556 & 465 & $84 \%$ & 1.95 & $8.51 \pm 1.62$ & $8.79 \pm 1.64$ \\
\hline \multicolumn{7}{|c|}{ Weighted mean mass of (52) Europa: } & $\begin{array}{l}4.00 \pm 0.78 \\
2.65 \pm 0.88\end{array}$ & $\begin{array}{r}4.00 \pm 0.78 \\
\mathbf{2 . 6 1} \pm \mathbf{0 . 8 8}\end{array}$ \\
\hline
\end{tabular}

(88) Thisbe

(7) Iris

1952 Dec. 28

0.017

$2.1 \quad 1848-1997$

8110

$7207 \quad 89 \% \quad 0.63$

$1.16 \pm 0.13$

$0.74 \pm 0.13$

\section{(444) Gyptis}

(54) Alexandra 1974 Nov. $02 \quad 0.002 \quad 3.2 \quad 1863-1998 \quad 586$

$457 \quad 78 \% \quad 1.58$

$0.42 \pm 0.16$

$0.36 \pm 0.16$

\begin{tabular}{lcccccccccc} 
(532) Herculina & 1963 Apr. 04 & 0.031 & 3.2 & $1904-1997$ & 3744 & 3266 & $87 \%$ & 0.59 & $3.52 \pm 0.29$ & $3.49 \pm 0.29$ \\
(89) Julia & 1957 Oct. 27 & 0.039 & 1.0 & $1866-1998$ & 1240 & 1071 & $87 \%$ & 1.15 & $3.93 \pm 1.37$ & $0.10 \pm 1.34$ \\
& & \multicolumn{4}{c}{ Weighted mean mass of (511) Davida: } & $3.54 \pm 0.28$ & $\mathbf{3 . 3 4} \pm \mathbf{0 . 2 8}$
\end{tabular}

\section{(511) Davida}

(704) Interamnia

\begin{tabular}{|c|c|c|c|c|c|c|c|c|c|c|}
\hline (95) Arethusa & 1985 Mar. 13 & 0.144 & 5.9 & $1872-1998$ & 492 & 342 & $70 \%$ & 1.67 & $3.00 \pm 1.26$ & $4.36 \pm 1.22$ \\
\hline (37) Fides & 1957 Aug. 19 & 0.057 & 2.3 & $1856-1998$ & 1544 & 1279 & $83 \%$ & 1.46 & $4.11 \pm 1.54$ & $6.47 \pm 1.46$ \\
\hline (993) Moultona & 1973 Nov. 23 & 0.014 & 1.5 & $1928-1998$ & 314 & 278 & $89 \%$ & 1.29 & $4.39 \pm 3.26$ & $4.39 \pm 3.26$ \\
\hline \multicolumn{9}{|c|}{ Weighted mean mass of (704) Intera } & $3.52 \pm 0.93$ & $5.16 \pm 0.90$ \\
\hline
\end{tabular}


Table 3. Standard deviations of post-fit, post-selection residuals for all of the test asteroids (given in boldface). The residuals were calculated for the standard dynamical model. In the table are given: number of observations: initial (before improvement) and final (after improvement and selection, separately in right ascension and declination); standard deviations in each block. Details of residuals for (7) Iris are taken from determinations of mass of (10) Hygiea; for (15) Eunomia and (88) Thisbe the standard deviations of (7) Iris residuals are very close. The standard deviations for (993) Moultona are exactly the same for both (52) Europa and (704) Interamnia.

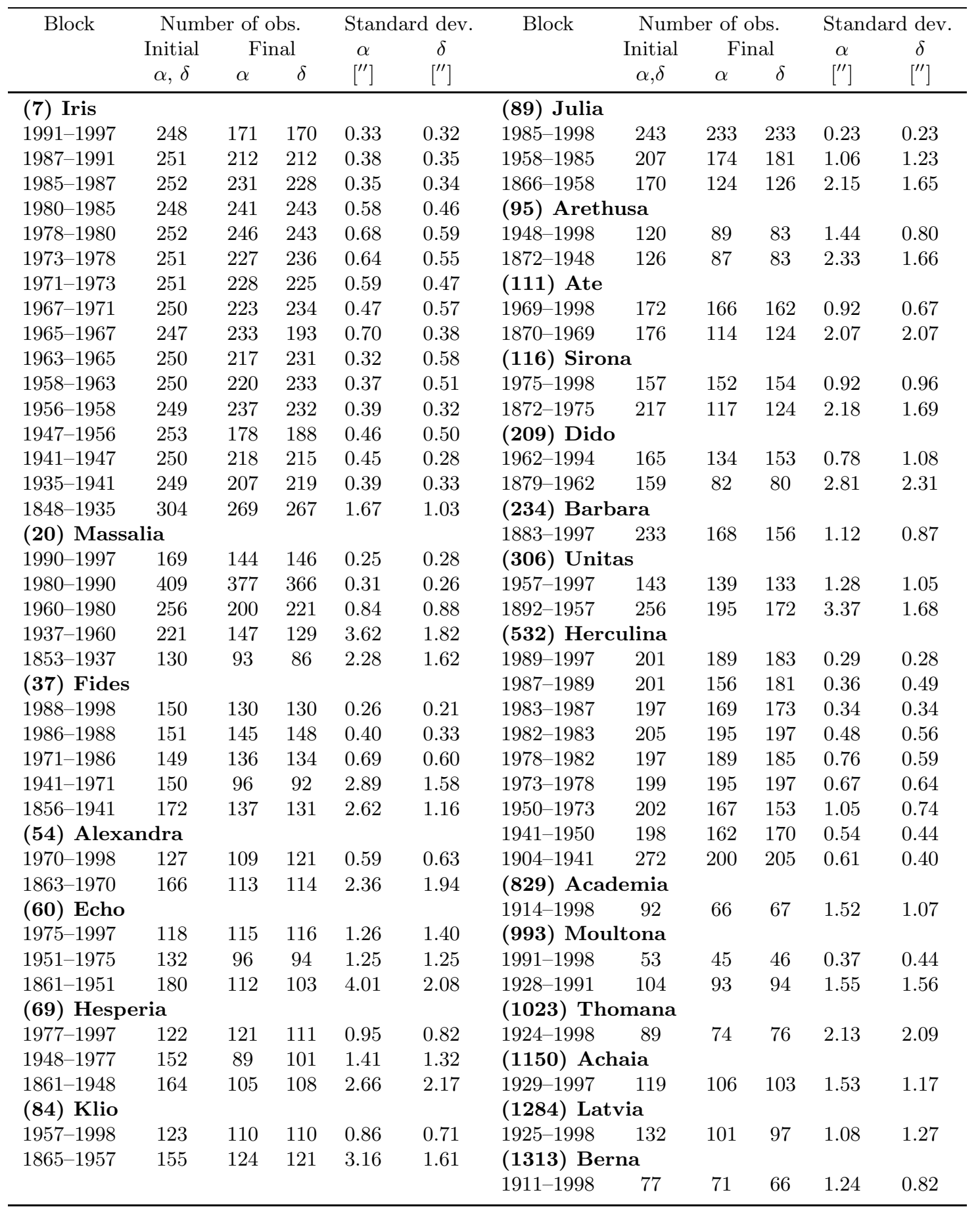

the adjusted masses were assumed to be those calculated from their diameter and a density of $3 \mathrm{~g} \mathrm{~cm}^{-3}$ (Table 6): $0.24 \times 10^{-10} M_{\odot}$ and $0.30 \times 10^{-10} M_{\odot}$, respectively, and this is denoted in Table 4 by an asterisk followed by the asteroid number.

Next, retaining values of masses as in the standard dynamical model (Table 1), single additional perturbing asteroids were added to the dynamical model. For example: in Table 4 "STD+(15)" means that a mass was calculated using the standard dynamical model supplemented with (15) Eunomia.

Finally, the asteroid masses were calculated for dynamical models including both the asteroids from the standard dynamical model and all additional perturbing asteroids 
Table 5. The asteroid densities calculated for final masses given in boldface in Table 2 .

\begin{tabular}{llll}
\hline Asteroid & Class $\begin{array}{l}\text { Diameter } \\
{[\mathrm{km}]}\end{array}$ & $\begin{array}{l}\text { Desity } \\
{\left[\mathrm{g} \mathrm{cm}^{-3}\right]}\end{array}$ \\
\hline (6) Hebe & $\mathrm{S}$ & $192 \pm 4^{1}$ & $3.7 \pm 1.2$ \\
& & $195 \pm 6 \times 170 \pm 6^{5}$ & $4.1 \pm 1.3$ \\
(10) Hygiea & $\mathrm{C}$ & $429 \pm 8^{1}$ & $2.7 \pm 0.4$ \\
& & $444 \pm 35^{2}$ & $2.4 \pm 0.6$ \\
(15) Eunomia & $\mathrm{S}$ & $272 \pm 6^{1}$ & $2.4 \pm 0.6$ \\
& & $305 \pm 22^{2 a}$ & $1.7 \pm 0.5$ \\
& & $370 \times 240^{2 b}$ & $3.2 \pm 1.7$ \\
& & $240 \times 60^{2 c}$ & $5.5 \pm 2.9$ \\
(52) Europa & $\mathrm{C}$ & $312 \pm 7^{1}$ & $3.3 \pm 1.1$ \\
(88) Thisbe & $\mathrm{C}$ & $214 \pm 32^{3}$ & $2.9 \pm 1.4$ \\
& & $232 \pm 12^{4}$ & $2.3 \pm 0.5$ \\
(444) Gyptis & $\mathrm{C}$ & $170 \pm 10^{1}$ & $2.8 \pm 1.3$ \\
(511) Davida & $\mathrm{C}$ & $337 \pm 5^{1}$ & $3.3 \pm 0.3$ \\
(704) Interamnia & $\mathrm{F}$ & $333 \pm 66^{1}$ & $3.6 \pm 1.0$ \\
\hline
\end{tabular}

References:

1 Tedesco (1989).

${ }^{2}$ Ragazzoni et al. (2000), ${ }^{2 a}$ spherical shape, ${ }^{2 b}$ egg-shaped,

${ }^{2 c}$ Binary model.

3 Bowell et al. (1979).

${ }^{4}$ Millis et al. (1983).

${ }^{5}$ Taylor \& Dunham (1978).

given in Table 7 ("STD+ALL" in Table 4). These models will be referred to as the extended dynamical models, dependent on a test asteroid. Asteroid masses in these models are taken from Table 6 and one should realize that they can be erroneous.

Asteroid densities, given in Table 5, were calculated from final masses (given in boldface in Table 2) for diameters found in the literature.

We shall now give some details and discuss the individual mass determinations.

\section{1. (6) Hebe}

Fourteen test asteroids were initially selected for determination of the mass of (6) Hebe. After the determination of the mass for all of them, we finally accepted only two solutions: for (234) Barbara and (1150) Achaia. They enabled us to determine the mass of Hebe for the first time. Comparison of the mean masses found for the standard and extended dynamical models: $(0.67 \pm 0.23) \times 10^{-11} M_{\odot}$ and $(0.69 \pm 0.22) \times 10^{-11} M_{\odot}$, respectively, shows, that this value is practically the same in both cases.

We finally accepted the mean mass obtained for the extended models since it was calculated with more real dynamical models. The mean density of Hebe (S-class, see Table 5) is $3.7 \pm 1.2 \mathrm{~g} \mathrm{~cm}^{-3}$ for the diameter from Tedesco (1989) or $4.1 \pm 1.3 \mathrm{~g} \mathrm{~cm}^{-3}$ for the diameter from a stellar occultation (Taylor \& Dunham 1978).

As can be seen in Fig. 1, where details of the perturbation of Hebe on both test asteroids are given, Achaia had a single, very close encounter with Hebe in 1943, while
Table 6. Assumed masses of perturbing asteroids used in calculations of perturbations of the test asteroids. All masses are expressed in $10^{-10} M_{\odot}$.

\begin{tabular}{llllll}
\hline $\begin{array}{l}\text { Asteroid } \\
\text { number }\end{array}$ & Mass & $\begin{array}{l}\text { Asteroid } \\
\text { number }\end{array}$ & Mass & $\begin{array}{l}\text { Asteroid } \\
\text { number }\end{array}$ & Mass \\
\hline$(1)$ & 4.7 & $(39)$ & 0.032 & $(150)$ & 0.031 \\
$(2)$ & 1.21 & $(41)$ & 0.047 & $(154)$ & 0.056 \\
$(4)$ & 1.36 & $(44)$ & 0.0031 & $(165)$ & 0.032 \\
$(5)$ & 0.015 & $(45)$ & 0.077 & $(194)$ & 0.042 \\
$(6)$ & 0.072 & $(46)$ & 0.018 & $(211)$ & 0.026 \\
$(7)$ & 0.050 & $(48)$ & 0.090 & $(230)$ & 0.011 \\
$(8)$ & 0.022 & $(51)$ & 0.028 & $(324)$ & 0.11 \\
$(9)$ & 0.037 & $(52)$ & 0.24 & $(356)$ & 0.019 \\
$(10)$ & 0.47 & $(56)$ & 0.013 & $(387)$ & 0.0094 \\
$(13)$ & 0.079 & $(59)$ & 0.041 & $(423)$ & 0.081 \\
$(15)$ & 0.16 & $(81)$ & 0.015 & $(444)$ & 0.039 \\
$(16)$ & 0.14 & $(88)$ & 0.077 & $(511)$ & 0.30 \\
$(19)$ & 0.06 & $(89)$ & 0.032 & $(532)$ & 0.097 \\
$(20)$ & 0.024 & $(91)$ & 0.012 & $(554)$ & 0.0075 \\
$(21)$ & 0.0077 & $(94)$ & 0.075 & $(704)$ & 0.35 \\
$(24)$ & 0.12 & $(96)$ & 0.042 & $(712)$ & 0.018 \\
$(27)$ & 0.013 & $(120)$ & 0.045 & & \\
$(29)$ & 0.084 & $(128)$ & 0.058 & & \\
\hline
\end{tabular}

Barbara underwent two more recent encounters, in 1946 and 1964. Therefore, the result obtained from Achaia relies on its oldest observations made over 14 years before the encounter in 1943. The situation is better for Barbara - its observations cover a period of 63 years before the closest encounter in 1946.

Looking at Table 7, where dynamical models for these two test asteroids are proposed, it can be seen that Achaia is perturbed by a smaller number of asteroids compared to Barbara. It can be seen from Table 4 that in the case of Achaia, only (15) Eunomia can be an important perturber and the mass of Hebe is not sensitive to errors in the masses of (1) Ceres and (4) Vesta. It can be seen from this table that for Barbara three additional asteroids can be important: (48) Doris, (194) Prokne and (387) Aquitania, which changes the mass of Hebe significantly. It can be also noticed in this case that erroneous masses of Ceres, Pallas and Vesta in the standard model can influence the mass of Hebe in a non-negligible way. Reliable estimation of the mass of Hebe from Barbara requires improvement of all masses in the dynamical model.

\section{2. (10) Hygiea}

From 38 initially selected candidates for the determination of the mass of Hygiea, we finally accepted 8 with the smallest standard deviations of the mass (see Table 2). There is a large (exceeding $4 \sigma$ ) difference between the weighted mean mass obtained for the standard and the extended dynamical models $\left((5.21 \pm 0.50) \times 10^{-11} M_{\odot}\right.$ and $(7.35 \pm 0.48) \times 10^{-11} M_{\odot}$ respectively). This is due to the fact that the result obtained from (7) Iris differs significantly for both models and, having the smallest 
formal error, remarkably influences the mean. It can be seen from Tables 7 and 4 that many asteroids influence the orbit of Iris. The most important are (15) Eunomia, (88) Thisbe and (89) Julia which give, when included in the dynamical model, very large differences in the mass of Hygiea not only in comparison with the standard deviation $(2.27 \sigma, 3.80 \sigma, 1.46 \sigma$ respectively), but also in relative value, reaching $30 \%, 50 \%$ and $20 \%$ of the mass obtained for the standard model. The mass of Hygiea obtained from Iris for the extended model is almost twice as large as for the standard model. A more reliable result from Iris requires not only a simultaneous solution for the mass of Hygiea, Eunomia and Thisbe (high correlation coefficients between the masses are expected that can prevent one from obtaining a unique solution), but also inclusion of correct masses for all asteroids listed in Table 7. Moreover, the residuals in right ascension of the oldest observations of Iris (before 1880) show a systematic drift of $1.4^{\prime \prime}$. This shift is the same in the case of determination of masses of (15) Eunomia and (88) Thisbe (Fig. 1) and also is present when the extended dynamical models are used.

For the reasons given above we decided to neglect the solution obtained from Iris. The weighted mean of the remaining 7 individual results is not significantly affected by changes in the dynamical model. The finally accepted mass of Hygiea, $(5.57 \pm 0.70) \times 10^{-11} M_{\odot}$ (the extended dynamical models $)$ is close to $(4.7 \pm 2.3) \times 10^{-11} M_{\odot}$, the mass obtained by Scholl et al. (1987), but has a significantly smaller formal error.

Assuming the IRAS diameter of this asteroid (Tedesco 1989, see Table 5), we obtain its mean density (C-class asteroid) of $2.7 \pm 0.4 \mathrm{~g} \mathrm{~cm}^{-3}$. When the diameter obtained from speckle interferometry (Ragazzoni et al. 2000) is assumed, the mean density is lowered to $2.4 \pm 0.6 \mathrm{~g} \mathrm{~cm}^{-3}$.

It can be seen from Table 4 that (829) Academia is perturbed by a very small number of asteroids and is potentially the best candidate for determination of the mass of Hygiea. In our database there are, however, very few pre-encounter observations of Academia. Collecting additional ones not included in MPC database would be highly desirable for more reliable determination of Hygiea's mass.

\section{3. (15) Eunomia}

An attempt to determine the mass of (15) Eunomia was first undertaken by Hilton (1997) from perturbations on (1284) Latvia and (1313) Berna. His mass, however, (4.2士 1.1) $\times 10^{-12} M_{\odot}$, derived from perturbation on (1313) Berna, gives a very small density for Eunomia, namely $0.79 \pm 0.21 \mathrm{~g} \mathrm{~cm}^{-3}$.

After examination of 11 candidates we finally accepted 3 solutions: for (7) Iris, (1284) Latvia and (1313) Berna (see Table 2). By comparison of our results with those obtained by Hilton (1997), it can be seen that in our case (1284) Latvia is the best test asteroid and gives the smallest formal error. Moreover, our mass of Eunomia determined from this test asteroid is three times larger than Hilton's mass.

Examination of dynamical models for the three test asteroids (Table 7 ) shows that Latvia is perturbed only by three minor planets: Ceres, Vesta and Eunomia. Moreover, the result is not sensitive to changes in masses of Ceres and Vesta within their errors (see Table 4). For this reason, Eunomia's mass found from this test asteroid, $(1.25 \pm 0.34) \times 10^{-11} M_{\odot}$, appears to be reliable.

The same reasoning can be applied to the result obtained from Berna: both changes in the mass of Ceres and inclusion of (165) Lorely and (230) Athamantis as additional perturbing asteroids (Table 4) have negligible effect on the mass of Eunomia.

The result obtained from (7) Iris, in spite of the small formal error, cannot be regarded as reliable due to its large sensitivity to perturbations from many other asteroids (see comments on this asteroid in the case of Hygiea in Sect. 2.2). For the extended dynamical model we obtained a negative value for the mass of Eunomia.

The mass of Eunomia was therefore calculated without the solution obtained from Iris, for the extended dynamical models, and equals $(1.26 \pm 0.30) \times 10^{-11} M_{\odot}$.

The densities, calculated for different diameters and shapes of Eunomia, are given in Table 5. For Tedesco's (1989) diameter we obtain a mean density of $2.4 \pm$ $0.6 \mathrm{~g} \mathrm{~cm}^{-3}$. Assuming speckle interferometry diameters and shapes (Ragazzoni et al. 2000), it can be seen that for a spherical shape and the binary asteroid model, the densities are very low $\left(1.7 \pm 0.5 \mathrm{~g} \mathrm{~cm}^{-3}\right)$ and very high $\left(5.5 \pm 2.9 \mathrm{~g} \mathrm{~cm}^{-3}\right)$, respectively. This suggests that the binary asteroid model may be inappropriate. For the eggshaped model, the density is $3.2 \pm 1.7 \mathrm{~g} \mathrm{~cm}^{-3}$ and this model appears to be more appropriate. Moreover, the light curve for Eunomia exhibits about $0.5 \mathrm{mag}$ variation (Cellino at al. 1985), which means that the asteroid is probably ellipsoidal in shape. In addition, the recent adaptive optics observations of Eunomia confirm the elongated shape of the asteroid and exclude its duplicity (Dumas 1999).

\section{4. (52) Europa}

(52) Europa is one of the asteroids used in our standard dynamical model, but for which there was no direct mass determination yet published. Only recently was its mass determined, by Kochetova \& Charnetenko (2000), from perturbations on asteroids being in a 2:1 commensurability with Jupiter. They obtained the value of $(2.9 \pm 1.6) \times 10^{-11} M_{\odot}$.

Our search gave 17 potentially useful asteroids for the determination of the mass of Europa. We finally accepted 4 solutions with smallest errors: from (84) Klio, (306) Unitas, (993) Moultona and (1023) Thomana. It can be seen from Table 2 that there are not significant differences between the masses obtained for the standard and the extended dynamical models. The weighted 
mean of all solutions (the extended models) amounts to $(4.00 \pm 0.78) \times 10^{-11} M_{\odot}$. This value is almost three times larger than the value $1.4 \times 10^{-11} M_{\odot}$ used in our standard dynamical model, that is estimated from a diameter of $312 \mathrm{~km}$ and a density of $1.8 \mathrm{~g} \mathrm{~cm}^{-3}$. The mass of Europa obtained from Klio as the test asteroid is very high. Europa's density for this case is $10.6 \mathrm{~g} \mathrm{~cm}^{-3}$ and we decided to exclude this result from the weighted mean. The next argument for the rejecting of this solution is that the year of the closest encounter between Klio and Europa, 1994, is very recent and future observations of Klio are required. But, as can be seen in Fig. 1, where details of perturbations of Europa on Klio are given, the perturbations are generated not only by the most recent encounter, but also by a series of older, more distant encounters. Moreover, Klio had a close encounter with Ceres in 1990 that resulted in a very large $\left(55^{\prime \prime}\right.$ in right ascension) perturbation effect. However, inspection of the dynamical models for Klio in Table 4 shows that there is no perturber capable of decreasing the mass of Europa. The most probable reason for this high mass is a systematic error in the observations or too short time span covered by the observations after the closest encounter in 1994.

The final value of mass of Europa is therefore calculated from the three remaining solutions and equals $(2.61 \pm 0.88) \times 10^{-11} M_{\odot}$ (the extended models). Its mean density determined for the IRAS diameter (Tedesco 1989) is $3.3 \pm 1.0 \mathrm{~g} \mathrm{~cm}^{-3}$.

From Tables 7 and 4 it can be seen that for asteroid (1023) Thomana, Europa is the only significant perturber; the influence of errors in the mass of Pallas is very small. The mass obtained from Thomana, however, has a large formal error. Collecting additional observations, both archival and future, can make this asteroid the best for determination of the mass of Europa.

In the case of (993) Moultona, the mass is strongly dependent on the mass of (704) Interamnia (see Table 4). Moreover, there are very few observations of this asteroid before the closest encounter with Europa in 1966 (see Fig. 1). Collecting additional archived observations of Moultona is crucial for reliable determination of Europa's mass.

\section{5. (88) Thisbe}

In the case of the C-class asteroid (88) Thisbe, only one of two candidates, (7) Iris, was accepted as a good test asteroid for determination of the mass.

As indicated above in the case of (10) Hygiea and (15) Eunomia (Sects. 2.2 and 2.3), Iris is strongly perturbed by many minor planets. Iris, however, is much more sensitive to perturbations from Thisbe than from Hygiea and Eunomia because Thisbe produces larger (than the two above) perturbation effect on Iris' orbit (see Table 7). Also the formal error of the mass of Thisbe obtained from Iris $\left(0.13 \times 10^{-11} M_{\odot}\right)$ is much smaller than for Hygiea and Eunomia (about $0.7 \times 10^{-11} M_{\odot}$ in both cases). In conse- quence, relative difference between the mass obtained for the extended dynamical model $\left((0.74 \pm 0.13) \times 10^{-11} M_{\odot}\right)$ and the standard one $\left((1.15 \pm 0.13) \times 10^{-11} M_{\odot}\right)$ is not very large (about 30\%). The mass of Thisbe obtained from Iris is not so drastically sensitive to the incompleteness of its dynamical model as in the case of (10) Hygiea and (15) Eunomia. For the latter two asteroids the differences between their masses for the standard and the extended dynamical models are much larger and reach about $100 \%$ and $200 \%$, respectively, in comparison to the masses for the standard model.

Finally we accept the mass of Thisbe, $(0.74 \pm 0.13) \times$ $10^{-11} M_{\odot}$, that is obtained for the extended, more real dynamical model. In spite of that, the mass of Thisbe should be treated as a first approximation only and can change even significantly after updating the dynamical model.

The mean density of the asteroid derived for its Tedesco (1989) diameter (see Table 5) is $2.9 \pm 1.4 \mathrm{~g} \mathrm{~cm}^{-3}$. The large formal error in the density is due to the large uncertainty in the diameter. For Thisbe, however, an additional estimation of the diameter from a stellar occultation is also avaliable (Millis et al. 1983). The density calculated for this diameter equals $2.3 \pm 0.5 \mathrm{~g} \mathrm{~cm}^{-3}$. Both estimates of the density appear to be quite acceptable.

\section{6. (444) Gyptis}

The mass of Gyptis, the C-class asteroid, was determined from perturbations exerted on one test asteroid, (54) Alexandra, which was found to be the only suitable candidate. The mass of Gyptis obtained for the exteded dynamical model amounts to $(3.6 \pm 1.6) \times 10^{-12} M_{\odot}$, which implies its mean density, assuming the Tedesco (1989) diameter, to be $2.8 \pm 1.3 \mathrm{~g} \mathrm{~cm}^{-3}$.

From Table 4 it can be seen that the mass of Gyptis is not sensitive to variations in masses of Ceres and Vesta while an incorrect mass of Hygiea can slightly influence the result. Relatively important in the dynamical model are the two additional asteroids (19) Fortuna and (45) Eugenia which, when added to the model, lower the resulting mass by over $30 \%$.

If the mass of Eugenia determined from its natural satellite (see Table 1 in Paper I) is correct, then its perturbation effect on Alexandra is about a half of that given in Table 7. This is a direct consequence of the two times smaller actual mass of Eugenia than that assumed in the calculation of the perturbation of Alexandra (see Table 7). This can result in a smaller influence of Eugenia on the mass of Gyptis obtained from Alexandra.

\section{7. (511) Davida}

The C-class asteroid (511) Davida is the second one used in our dynamical model for which the mass was estimated indirectly, from a mean diameter and a density for this asteroid class. The mass of Davida was also estimated by Kochetova \& Charnetenko (2000) from perturbations on 
asteroids being in a 2:1 commensurability with Jupiter. These authors obtained a very high value of the mass, $(7.9 \pm 2.3) \times 10^{-11} M_{\odot}$, that implies, for the Tedesco (1989) diameter, a rather unrealistic density of $(7.8 \pm 2.3) \mathrm{g} \mathrm{cm}^{-3}$.

From 17 candidates found for the determination of Davida's mass, only 2 solutions with the smallest formal errors were finally accepted: those for (532) Herculina and (89) Julia.

The mass found from Herculina, $(3.52 \pm 0.29) \times$ $10^{-11} M_{\odot}$, (the standard model) has a four times smaller formal error than the mass $(3.93 \pm 1.37) \times 10^{-11} M_{\odot}$ found from Julia and therefore dominates the weighted mean. Herculina is also the best test asteroid because it gives Davida's mass that is almost insensitive to errors in masses in the standard dynamical model and inclusion the only additional perturbing asteroid, (444) Gyptis, to the dynamical model (see Table 4). Moreover, Herculina was observed for a long enough time before and after the closest encounter with Davida in 1963 (Fig. 1), and the number of observations in both intervals is large. For these reasons the mass of Davida found from perturbations on Herculina can be treated as reliable, provided that the observations are not significantly biased by systematic errors.

The result obtained from (89) Julia, as opposed to Herculina, is strongly depedent on asteroid masses included in the dynamical model (Table 4). The influence of the errors in masses of Vesta and Hygiea as well as perturbations from other asteroids ((96) Aegle being the most important one) is significant. In spite of this, the result obtained from Julia is included in the weighted mean because, due to the large formal error, it does not significantly change the mean. Therefore, the final mass of Davida amounts to $(3.34 \pm 0.28) \times 10^{-11} M_{\odot}$ (the extended dynamical models). This value is almost twice as large as the value $1.8 \times 10^{-11} M_{\odot}$, i.e. the mass used in our dynamical model.

Assuming the IRAS dimeter (Tedesco 1989) of Davida, its mean density is $3.3 \pm 0.3 \mathrm{~g} \mathrm{~cm}^{-3}$.

\section{8. (704) Interamnia}

The only mass determination of Interamnia found in the literature is that made by Landgraf (1992) from perturbations on (993) Moultona. The result, $(3.7 \pm 1.7) \times$ $10^{-11} M_{\odot}$, was obtained, however, without taking into account any additional asteroidal perturbations, and from observations made before 1991.

From 13 candidate test asteroids found, we accepted three of them: (37) Fides, (95) Arethusa and (993) Moultona.

It can be seen from Table 2 that the mean mass of Interamnia obtained for the extended dynamical models, $(5.16 \pm 0.90) \times 10^{-11} M_{\odot}$, is significantly higher than $(3.52 \pm 0.93) \times 10^{-11} M_{\odot}$, the mean value found for the standard model. The former mass implies a very high density for Intermnia, a value of $5.3 \mathrm{~g} \mathrm{~cm}^{-3}$.
The asteroid for which the highest mass was obtained when using the extended model is (37) Fides. It can be seen in Table 7 that Fides is strongly perturbed by many asteroids and the largest changes in the mass of Interamnia are introduced by inclusion of (19) Fortuna and (29) Amphitrite to the dynamical model. One of the sources of this large discrepancy between the result obtained using the standard and the extended model can be that the masses of the additional asteroids in the extended dynamical model, estimated from diameters and a density of $3 \mathrm{~g} \mathrm{~cm}^{-3}$, can be wrong. Another source is possible systematic errors in the oldest observations of Fides. It can be seen in Fig. 1, where the residuals of Fides are plotted, that in the oldest group of observations in right ascension (before 1880), there is an evident drift of about $2^{\prime \prime}$. Although the residuals are calculated for the standard dynamical model, the shift is almost exactly the same for the extended dynamical model.

Also, higher mass was obtained from (95) Arethusa when using the extended model. In this case, two factors can be responsible. First, the assumed mass of (211) Isolda and (16) Psyche can be too high. Second, Arethusa had a series of very recent encounters with Interamnia. The closest one was in 1985 (see Fig. 1 for details of the perturbations) and the time span covered by the observations of Arethusa after the encounter is too short. Further observations of this asteroid are highly desirable and can enable a more exact and reliable estimation of Interamnia's mass.

The mass of Interamnia obtained from (993) Moultona has a very large formal error and old pre-encounter observations of the asteroid are needed to decrease the uncertainty.

For the reasons given above, in this paper we finally decided to accept the mean mass $(3.52 \pm 0.93) \times 10^{-11} M_{\odot}$ obtained for the standard dynamical model, having in mind that this mass was obtained assuming an incomplete dynamical model.

A mean density of Interamnia, assuming its IRAS diameter, amounts to $3.6 \pm 1.0 \mathrm{~g} \mathrm{~cm}^{-3}$.

\section{Summary and conclusions}

In this second paper of the series, new masses for eight asteroids were found. For five of them (i.e. (10) Hygiea, (15) Eunomia, (52) Europa, (511) Davida and (704) Interamnia) the masses obtained are an improvement of the values found by other authors. The masses of (6) Hebe, (88) Thisbe and (444) Gyptis were determined for the first time. Compared to previous estimations, the most different mass is that of Eunomia, for which we obtained a three times larger value than that found by Hilton (1997). The masses of Europa and Davida appeared to be about two times larger than those used in our standard dynamical model.

Due to large formal errors in the mean densities and the small sample of asteroids, no dependence between asteroid densities and asteroid class is visible. The weighted mean density of $\mathrm{C}$-class asteroids (five objects, 
see Table 5) equals $2.9 \pm 0.2 \mathrm{~g} \mathrm{~cm}^{-3}$ and is significantly higher than $1.8 \mathrm{~g} \mathrm{~cm}^{-3}$, the value assumed for this class in DE405 ephemerides. The weighted mean density of Sclass asteroids (two minor planets, without the spherical and the binary model for Eunomia) amounts to $2.9 \pm 0.5 \mathrm{~g} \mathrm{~cm}^{-3}$ and agrees within its error with the value of $2.4 \mathrm{~g} \mathrm{~cm}^{-3}$ assumed in these ephemerides.

All of the above asteroid masses can be, however, determined more reliably in the future if the dynamical models involved will be improved. For all test asteroids we investigated the influence of both errors in the masses included in the standard dynamical model and the influence of inclusion of additional perturbing asteroids to the dynamical model on derived masses, to indicate the most important perturbers. Finally accepted asteroid masses were calculated for the extended dynamical models (except for Interamnia). It should be remembered, however, that the masses of additional perturbing asteroids in these dynamical modeles are only estimates; they can be erroneous and introduce a bias in the derived masses.

The method of mass determinations used in this paper consists of calculating individual masses from perturbations on single test asteroids and then calculating the weighted mean. One can argue that the best method for the determination of asteroid masses is simulataneous determination from perturbations on many test asteroids. But if there is no correlation between the individual determinations, then both methods are equivalent (Viateau \& Rapaport 1998). In this paper, the asteroid (7) Iris could be used in simultaneous determinations of the mass of three asteroids: (10) Hygiea, (15) Eunomia and (88) Thisbe, but its orbit is perturbed significantly by many other asteroids and it is now too early to obtain a reliable solution by this method (additionaly, expected high correlation coefficients between the masses can disable a reliable and unique solution). Moreover, the masses presented here are obtained from sets of observations included only in MPC databases which are sometimes far from being complete. For these reasons, some of the weighted mean masses finally accepted in this paper can be misleading; more reliable values can be obtained after correcting of asteroid masses in the dynamical models and collecting additional observations.

This series of papers is designed to give first approximations of asteroid masses and find correct dynamical models for each of the test asteroids. This should be a good base for future, complete determinations of asteroid masses. For all of the test asteroids used, collection of additional both old (not included in the MPC database) and future observations would be very useful.

The need for additional old observations can be easily inferred from Fig. 1, where for the test asteroids the dates of the closest encounter and the distribution of observations used (filled circles) are clearly seen. The old observations are most important for asteroids (1150) Achaia (for the mass of (6) Hebe), (829) Academia (for the mass of (10) Hygiea) and (993) Moultona (for the mass of both (52) Europa and (704) Interamnia). On the other hand, further observations will be required for asteroids (111) Ate and (209) Dido (for the mass of (10) Hygiea), (84) Klio (for the mass of (52) Europa), (54) Alexandra (for the mass of (444) Gyptis) and (95) Arethusa (for the mass of (704) Interamnia).

Acknowledgements. I would like to thank Dr. A. Pigulski for critical reading of the paper.

\section{References}

Bowell, E., Gehrels, T., \& Zellner, B. 1979, Magnitudes, colors, types and adopted diameters of the asteroids, in Asteroids, ed. T. Gehrels (University of Arizona Press), 1108

Cellino, A., Pannunzio, R., Zappala, V., et al. 1985, A\&A, 144, 355

Dumas, C. 1999, private communication to Ragazzoni et al. (2000)

Hilton, J. L. 1997, AJ, 114, 402

Kochetova, O. M., \& Chernetenko, Y. A. 2000, in US-European Celestial Mechanics Workshop, 3-7 July 2000, Poznań, Poland, Poster No. 26

Landgraf, W. 1992, in Proc. IAU Symp. 152, Chaos, resonance and collective dynamical phenomena in the Solar System, ed. S. Ferraz-Mello (Kluwer, Dordrecht), 179

Michalak, G. 2000, A\&A, 360, 363, Paper I

Millis, R. L., Wasserman, O. G., Franz, N. M., et al. 1983, AJ, 88,229

Ragazzoni, R., Baruffolo, A., Marchetti, E., et al. 2000, A\&A, 354,315

Scholl, H., Schmadel, D., \& Roser, S. 1987, A\&A, 179, 311

Taylor, G. E., \& Dunham, D. W. 1978, Icarus, 34, 89

Tedesco, E. F. 1989, in Asteroids II, ed. R. P. Binzel, T. Gehrels, \& M. S. Matthews (University of Arizona Press), 1090

Tedesco, E. F. (ed.) 1992, The IRAS Minor planet survey. Tech. Rep. PL-TR-92-2049, Phillips Laboratory, Hanscom, AF Base, MA

Viateau, B., \& Rapaport, M. 1998, A\&A, 334, 729 